\title{
Growth and Nanofabrication of All-Perovskite Superconducting/Ferromagnetic/Superconducting Junctions
}

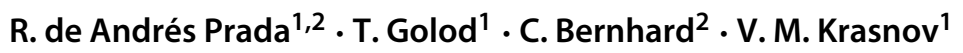 \\ Received: 14 May 2018 / Accepted: 16 January 2019 / Published online: 12 March 2019 \\ (C) The Author(s) 2019
}

\begin{abstract}
We fabricate and study experimentally all-perovskite-oxide superconductor/ferromagnetic insulator/superconductor (S/FI/S) tunnel junctions made out of the high-temperature cuprate superconductor $\mathrm{YBa}_{2} \mathrm{Cu}_{3} \mathrm{O}_{7-y}$ (YBCO) and the colossal magnetoresistive manganite $\mathrm{LaMnO}_{3}(\mathrm{LMO})$ in the ferromagnetic insulator state. YBCO/LMO/YBCO heterostructures with different LMO thicknesses $(5,10$, and $20 \mathrm{~nm})$ are grown epitaxially via pulsed laser deposition. Nanoscale S/FI/S junctions with sizes down to $300 \mathrm{~nm}$ are made by three-dimensional nano-sculpturing with focused ion beam. Junctions with a thick $(20 \mathrm{~nm}) \mathrm{LMO}$ barrier exhibit a large negative magnetoresistance below $T_{\text {Curie }} \sim 160 \mathrm{~K}$, typical for colossal magnetoresistive manganites, as well as a kink in the current-voltage characteristics at large bias ( $V \sim 1-2$ Volts), attributed to Zener-type tunneling. However, they do not show a measurable Josephson current. On the contrary, junctions with the thinnest 5-nm LMO barrier exhibit a large supercurrent and no signs of magnetism. The latter may indicate the presence of pinholes due to thickness inhomogeneity and/or a $\sim 2 \mathrm{~nm}$ dead magnetic layer at the YBCO / LMO interface caused, e.g., by interdiffusion or strain. The junction with an intermediate 10-nm LMO barrier exhibited a desired S/FI/S junction behavior with significant negative magnetoresistance and signatures of a small Josephson current.
\end{abstract}

Keywords Perovskites · Josephson junction · Tunnel junction · LMO · YBCO

\section{Introduction}

The competition of spin-singlet superconductivity and magnetism in superconductor/ferromagnet (SF) heterostructures leads to a variety of unusual physical phenomena, including possible generation of a long-range odd-frequency spintriplet component of the superconducting order parameter in the F-layers [1-6]. Those effects should be most pronounced and unambiguous in case of a fully spin-polarized ferromagnet, which does not support spin-singlet Cooper pairs. This is achievable with half-metallic ferromagnets such as $\mathrm{CrO}_{2}$ [7, 8], or colossal magnetoresistance manganites [9-14].

Here, we present an experimental study of transport and magnetic properties of nano-scale superconductor/ ferromagnetic insulator/superconductor (S/FI/S) junctions,

R. de Andrés Prada

roberto.deandres@fysik.su.se

1 Department of Physics, Stockholm University, Albanova University Center, SE-106 91, Stockholm, Sweden

2 Physics Department and Fribourg Center for Nanomaterials (FriMat), University of Fribourg, Chemin du Musée 3CH-1700, Fribourg, Switzerland composed exclusively of oxide materials with perovskitelike structure: a high- $T_{c}$ cuprate superconductor $\mathrm{YBa}_{2} \mathrm{Cu}_{3} \mathrm{O}_{7-y}$ (YBCO) with a superconducting critical temperature $\sim 90 \mathrm{~K}$, and a colossal magnetoresistance manganite $\mathrm{LaMnO}_{3}$ (LMO) in the FI state, which develops a spontaneous ferromagnetic momentum as it undergoes a metallic to insulating transition at the Curie temperature $T_{\text {Curie }} \sim 160 \mathrm{~K}$. The $\mathrm{YBCO} / \mathrm{LMO} / \mathrm{YBCO}$ trilayers with three different thicknesses for the FI layer $(20 \mathrm{~nm}, 10$ $\mathrm{nm}$, and $5 \mathrm{~nm}$ ) are deposited via pulsed laser deposition (PLD). Zigzag-type YBCO/LMO/YBCO junctions with micron-scale length and submicron width are made by microfabrication techniques and 3D nanosculpturing with focused ion beam (FIB). We analyze the temperature and magnetic field dependencies of the transport characteristics of these junctions.

\section{Experimental Procedures}

We study three YBCO (100 nm)/LMO (d)/YBCO (100 $\mathrm{nm})$ multilayers with different thicknesses $d$ of the FI barrier of $20 \mathrm{~nm}, 10 \mathrm{~nm}$, and $5 \mathrm{~nm}$. The YBCO layers are 
made thick in order to simplify the nanosculpturing with FIB. Measurements in a temperature range $1.7-300 \mathrm{~K}$ are performed in a closed cycle ${ }^{4} \mathrm{He}$ cryostat with sample-ingas cooling. The cryostat has a superconducting magnet with magnetic field up to $17 \mathrm{~T}$. The sample is placed on a rotating holder, which allows measurements of magnetic responses at different field orientations. Measurements are made in a quasi 4-probe configuration with separated wires for $I^{+} I^{-} V^{+} V^{-}$bonded to electrodes for each junction. At $T$ $>T_{c}$ of YBCO, it is only a quasi 4-probe because electrode resistances are added to the measurements. Below $T_{c}$, the electrodes become superconducting and the measurements become true 4-probe.

\subsection{Multilayer Deposition}

The YBCO/LMO/YBCO trilayers were grown via PLD using an excimer $\mathrm{KrF}$ laser $\left(\lambda=248 \mathrm{~nm}, t_{s}=25 \mathrm{~ns}\right)$ with a frequency and fluency of $2 \mathrm{~Hz}$ and $2 \mathrm{~J} \mathrm{~cm}^{-2}$, respectively. The laser footprint on the target had a size of $3 \times 1 \mathrm{~mm}^{2}$. Films are grown epitaxially on (001)-oriented $\mathrm{La}_{0.3} \mathrm{Sr}_{0.7} \mathrm{Al}_{0.65} \mathrm{Ta}_{0.35} \mathrm{O}_{3}$ (LSAT) substrates. During the growth, the substrates were maintained at $900{ }^{\circ} \mathrm{C}$ in a partial $\mathrm{O}_{2}$ pressure of 0.32 mbar. The deposition was monitored with in situ reflection high-energy electron diffraction (RHEED) using a collimated $30 \mathrm{kV}$ electron gun at low incidence angle. After the deposition, the samples were cooled to $700{ }^{\circ} \mathrm{C}$ at a rate of $10{ }^{\circ} \mathrm{C} \mathrm{min}^{-1}$ in the same pressure conditions, before the oxygen gas pressure was increased to $1 \mathrm{bar}$. Consequently, the samples were cooled to $485^{\circ} \mathrm{C}$ at $30^{\circ} \mathrm{C} \mathrm{min}^{-1}$, and annealed at this temperature for one hour before cooling them to room temperature. Finally, the trilayers were covered by a protective gold layer of 70 $\mathrm{nm}$, which was deposited by thermal evaporation. Details of the deposition can be found in Ref. [14]. Figure 1a shows the X-ray reflectivity (XRR) data obtained for a single layer of YBCO of $70 \mathrm{~nm}$ deposited under these conditions. The datapoints were fit to a curve (red line), giving an average surface roughness of $\sim 1.6 \mathrm{~nm}$. This rather low value of the surface roughness suggests that the thin LMO layers deposited as FI barriers are uniform even when deposited on top of thick layers of YBCO.

\subsection{Junction Fabrication}

First, twelve $\sim 6-\mu \mathrm{m}$-wide electrodes are patterned on a chip using photolithography and cryogenic reactive ion etching in Ar plasma. The low process temperature prevents oxygen out-diffusion from the films during etching. Next, narrow bridges of submicrometric width are made at certain locations by FIB. Figure $1 \mathrm{~b}$ represents a scanning electron microscope (SEM) image of one of the samples with 12 interconnected electrodes and nine patterned bridges.
Finally, two side cuts through the top and bottom YBCO layers, respectively, are made by FIB at a grazing angle. Figure 1c shows a SEM image of one of the studied junctions. During the measurements, the current is sent along the electrodes, which are selected depending on the junction to be measured. Since the side cuts interrupt the top and bottom YBCO layers, the current is forced to flow across the LMO layer. The zigzag current path in the junction is indicated schematically in Fig. 1d. This allows both probing of the perpendicular transport characteristics of the LMO layer and analyzing the Josephson current between the two YBCO layers in the S/FI/S junction [13].
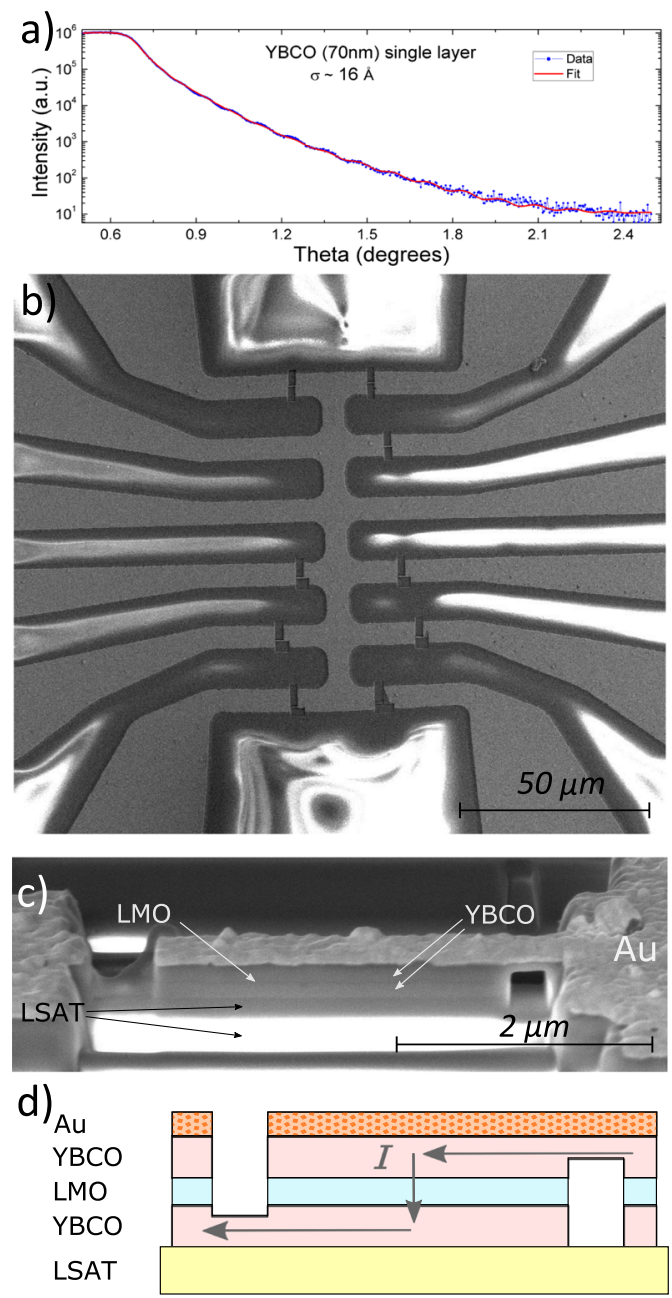

Fig. 1 a XRR datapoints and curve fit for a 70-nm-thick YBCO single layer. The surface roughness obtained from the fit is $\sim 1.6 \mathrm{~nm}$. YBCO/LMO/YBCO junction fabrication: $\mathbf{b}$ Top view of the sample with 12 electrodes and nine patterned nano-bridges. $\mathbf{c}$ SEM image of the junction No. 2a on the sample with 10-nm LMO. The top brighter film with uneven thickness is a protective Au layer of $70 \mathrm{~nm}$. The gray structure beneath it is the $\mathrm{YBCO} / \mathrm{LMO} / \mathrm{YBCO}$ heterostructure. The LMO layer can be seen as a darker line in the middle of the stack. The two side cuts at the right and the left sides of the bridge interrupt the top and the bottom YBCO layers, respectively. d Sketch of the side view of the junction (not in scale). Arrows indicate the zigzag current flow path 


\section{Results and Discussion}

\subsection{Junctions with 20-nm LMO}

Figure $2 \mathrm{a}$ shows the temperature dependence of the resistance measured with large $\mathrm{AC}$ current for one of the junctions with a 20 -nm-thick barrier at zero magnetic field. At the superconducting critical temperature of YBCO $T_{c} \simeq$ $90 \mathrm{~K}$, the resistance drops by few hundreds of ohms. The drop corresponds to the normal resistance of the $\mathrm{YBCO}$ layers in the electrodes, which is shown in the inset (for a bridge before side cuts). At $T<T_{c}$, the measured resistance represents solely the junction resistance. The strong upturn of resistance with decreasing $T$ confirms the insulating nature of the LMO layer in the junction. We note that for this junction, the upturn starts already from room temperature and the metal-insulator transition at $T_{\text {Curie }} \simeq 160 \mathrm{~K}$ is not
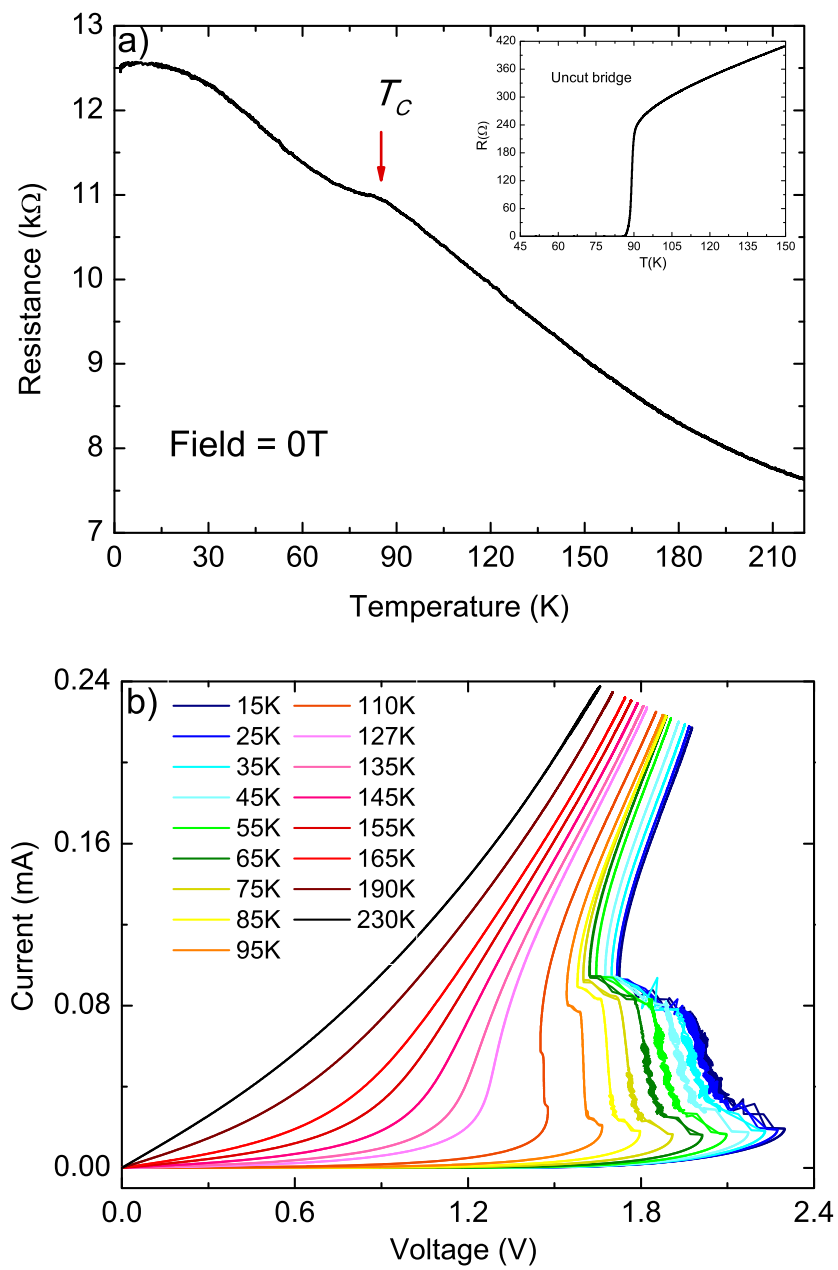

Fig. 2 20-nm LMO: Temperature dependencies at zero magnetic field. a Temperature dependence of large bias AC resistance for a YBCO/LMO $(20 \mathrm{~nm}) / \mathrm{YBCO}$ junction. The inset shows $R(T)$ for an uncut bridge, representing the in-plane resistance of YBCO layers. b Temperature evolution of $I-V$ characteristics for the same junction. The bias span is the same as for the measurement in panel a
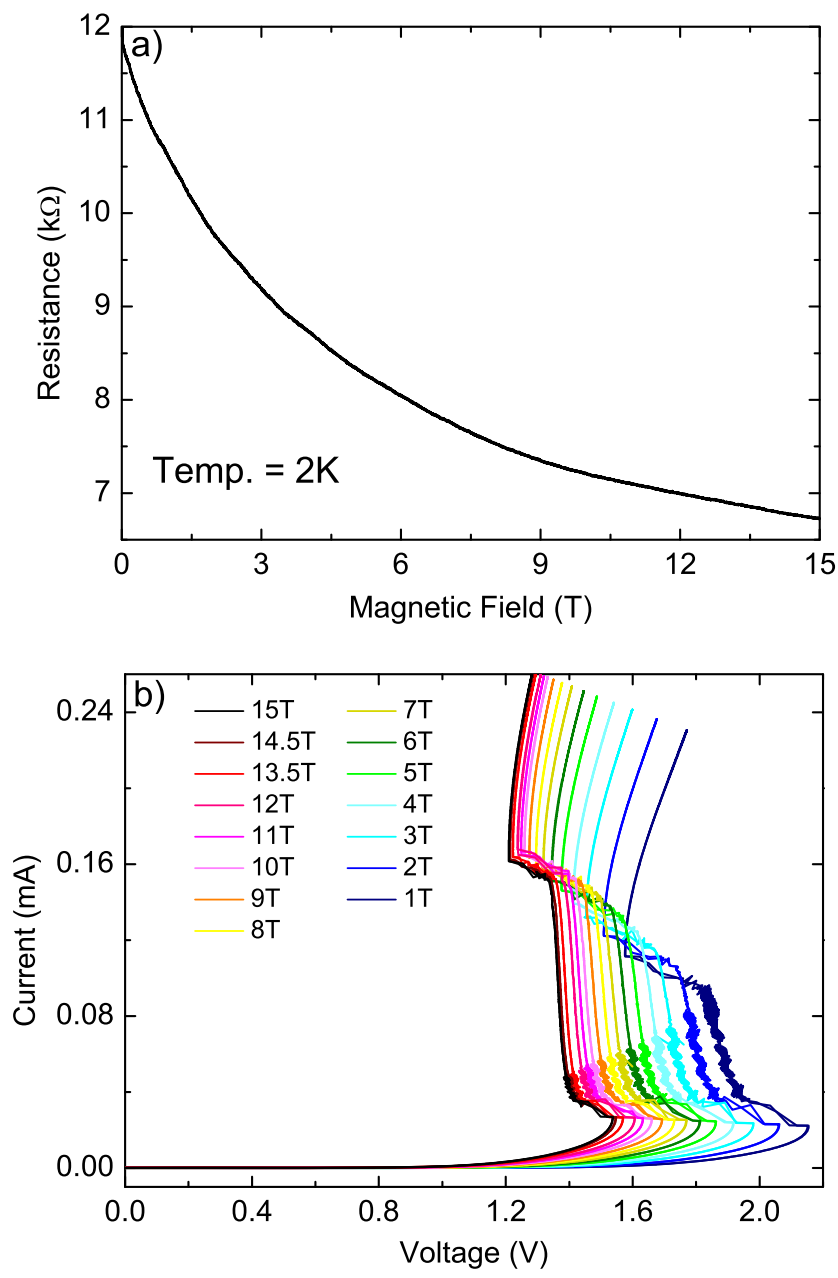

Fig. 3 20-nm LMO: Magnetic field dependencies at $T=2 \mathrm{~K}$. a Magnetoresistance of the same previous junction, measured at large AC-bias current. b Corresponding $I-V$ characteristics at different inplane fields

seen as on previously studied junctions at the same chip [13]. This might be caused by a partial passivation of the top YBCO layer during almost 3-year storage of the chip at ambient conditions, which becomes more underdoped (although still superconducting) and contributes to the measured resistance.

Figure $2 \mathrm{~b}$ shows current-voltage $(I-V)$ characteristics for the same junctions at different temperatures and at zero magnetic field. The amplitude of the current is also the same as in the $R(T)$ measurement shown in Fig. 2a. It is seen that the $I-V$ 's are nonlinear. As discussed in Ref. [13], the nonlinearity rapidly progresses below $T_{\text {Curie }} \simeq 160$ $\mathrm{K}$. Indeed, it is seen that at $T<160 \mathrm{~K}$, a pronounced kink appears in the $I-V$ 's, which develops into a negative differential resistance branch with two abrupt steps at low $T$. This feature has been studied in Ref. [13] and was attributed to Zener-type tunneling between spin-polarized conduction and valence bands in the LMO layer. Compared 

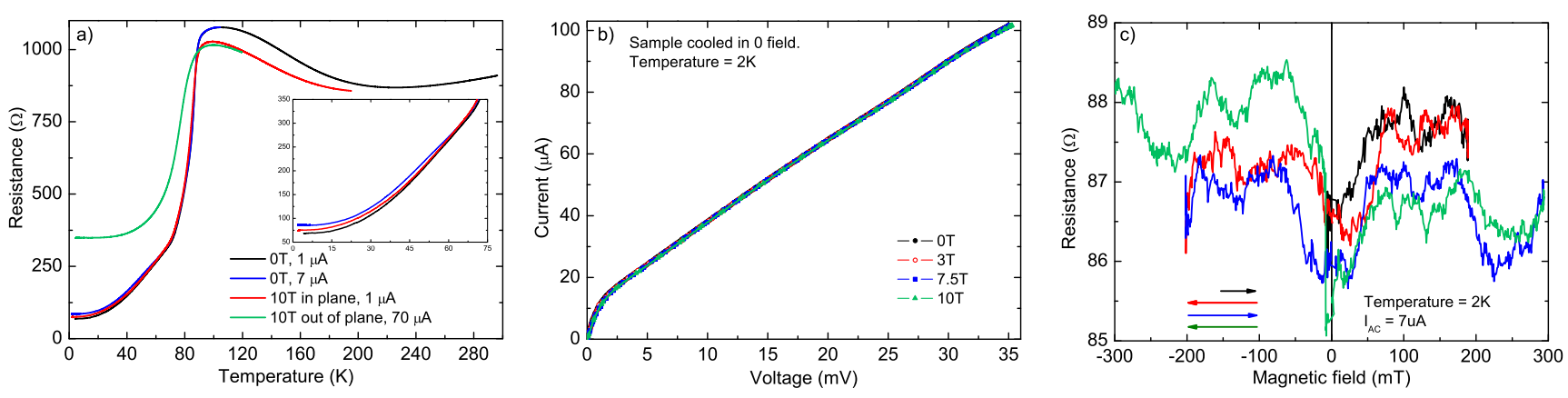

Fig. 4 Junctions with a 10-nm LMO barrier. a $R$ vs. $T$ of a junction at different field (and bias) conditions (inset-detail of the low temperature range). b $I$ - $V$ characteristics of the junction taken at different in-plane fields. $\mathbf{c}$ Low bias resistance versus in-plane field at $T=2 \mathrm{~K}$

with the previous report, the kink voltage is larger and increases monotonously with decreasing $T$. We attribute this difference to a lower doping state of YBCO layers and/or $\mathrm{YBCO} / \mathrm{LMO}$ interface in the present junction, which leads to an additional voltage drop with a strong insulating $T$ dependence, as seen from Fig. 2a.

Figure 3a shows magnetoresistance for the same junction in the in-plane magnetic field configuration at $T=2 \mathrm{~K}$. The measurement is made as in Fig. 2a. Figure 3b shows corresponding $I-V$ 's at different fields. A strong negative magnetoresistance, typical for colossal magnetoresistive manganites, is clearly seen. Thus, we observe both insulating behavior and magnetism in the 20-nm-thick LMO, confirming that it is indeed a FI at low $T$ [13]. However, no supercurrent is observed. This indicates that the 20-nm LMO is too thick and the barrier should be made significantly thinner for the occurrence of Josephson tunneling through the FI.

\subsection{Junctions with 10-nm LMO}

Figure 4 summarizes the characteristics of two different junctions with $10 \mathrm{~nm}$ of LMO. Panel a shows $R(T)$ for a junction. The key difference with respect to the junction with 20-nm-thick LMO (Fig. 2a), is a strong drop of resistance at $T_{c}$, indicating the occurrence of supercurrent through the junction. The overall value of $R$ is also strongly reduced (by an order of magnitude). This is expected for tunnel junctions, for which the resistance should decrease exponentially with decreasing the thickness of the barrier. For this junction, we can also clearly see the metal-insulator transition of LMO with decreasing $T$. In order to analyze the development of nonlinearity and magnetoresistance, we present $R(T)$ curves at different bias and fields. Comparison of the curves in black $(H=0 \mathrm{~T})$ and red $(H=10 \mathrm{~T}$ in plane), measured with the same $I_{a c}=1 \mu \mathrm{A}$, shows the appearance of a significant negative magnetoresistance in the insolating state which starts slightly higher than $160 \mathrm{~K}$. The inset in Fig. 4a shows in detail the low temperature range. A large bias in zero field (curve in blue) turns into a higher resistance than a low bias and high field (in red). The overall magnetic response of the 10-nm LMO is, therefore, much weaker than that of the 20-nm LMO. The curve in green $\left(I_{a c}\right.$ $=70 \mu \mathrm{A}, 10 \mathrm{~T}$ out-of-plane) shows the concomitance of a large bias with the penetration of vortices.

Figure $4 \mathrm{~b}$ shows $I-V$ curves at $T=2 \mathrm{~K}$ for different fields applied in plane. Signatures of a small Josephson current $I_{c} \sim 10 \mu \mathrm{A}$ are seen, although it is not developed into a perfect superconducting branch, which can be at least partly attributed to noise and fluctuations in our cryostat
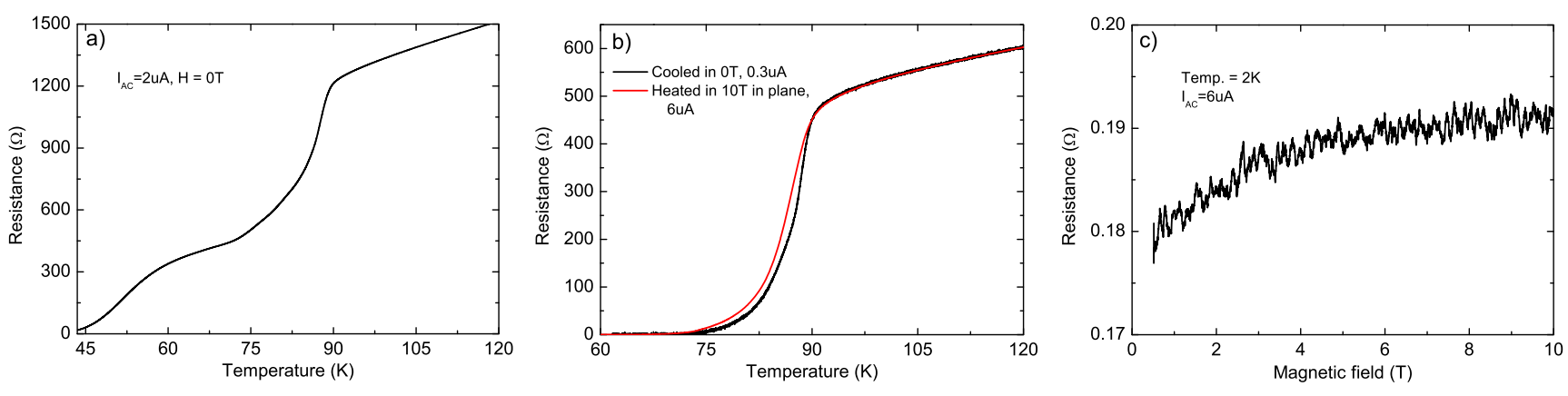

Fig. 5 Curves from devices fabricated on the sample with 5-nm-thick LMO. a Device No. 1a-resistance of the junction at $0 \mathrm{~T}$ as a function of temperature showing a SC transition in two steps. b Device No.
$2 \mathrm{a}-R(T)$ with a slight splitting during the SC transition as $10 \mathrm{~T}$ are applied in plane, and $\mathbf{c}$ the same device while the field is increased at low temperature 
without special shielding [15]. It is also seen that the I$V$ 's are not strongly affected by the magnetic field. Yet we do observe a small field modulation of the zero bias resistance, as demonstrated in Fig. 4c. Apparently, it is far from a clear/full Fraunhofer modulation. Most likely there are pinholes in the barrier, which deteriorate the quality of the junctions.

\subsection{Junctions with 5-nm LMO}

Five different junctions were fabricated and studied in the sample with 5-nm LMO, obtaining similar results in all of them. Figure $5 \mathrm{a}$ and $\mathrm{b}$ show $R(T)$ for two of these junctions. Here, we do not observe neither insulating behavior nor signs of negative magnetoresistance. Below $T_{c}$, the resistance of the junctions drops to zero. Panel c shows measured magnetoresistance for one of the junctions. It is positive, typical for superconductors. The supercurrent does not exhibit a Fraunhofer modulation. Therefore, it is not of Josephson type but is flowing, entirely or partially, through nano-scale shorts.

The magnetic nature of the barrier is difficult to determine, since we only observe its magnetic state indirectly via the perpendicular current, and this appears to be predominantly governed by pinholes. However, if the current was flowing entirely from YBCO to YBCO via pinholes, it would not see the barrier neither on the normal nor on the superconducting states of YBCO. This contrasts with the large resistances observed above $T_{C}$ which suggest that, to some extend, the current is influenced by the barrier. Should this be the case, the LMO does not show any relevant magnetic influence on the current.

Considering the presented data for junctions with different LMO thickness, it follows that the features of magnetism observed in the perpendicular current are weakened with decreasing thickness. Ferromagnetism in the 5-nm layer does not emerge, or does not do it with sufficient magnitude to influence the $R(H)$ curves. The presence of dead magnetic layers of $\sim 2-3 \mathrm{~nm}$ at interfaces would be consistent with a similar observation in Ref. [16] for $\mathrm{LaMnO}_{3} / \mathrm{SrTiO}_{3}$ heterostructures. Furthermore, the properties of manganites are known to be sensitive to mechanical stress and film structure [17-20], and defects originating from the thick bottom YBCO could also influence the development of a ferromagnetic moment. In this regard, adjusting the thickness of the bottom YBCO so it remains epitaxially strained could reduce the influence of defects originated from the thick bottom YBCO, which penetrate into the LMO barrier.

The origin of pinholes is unclear to us. Surface roughness is considered a decisive factor on the appearance of pinholes, but as shown in Fig. 1a, the YBCO surface is not rougher than 1 or 2 u.c. In a former work, we also demonstrated coherent interfaces over long lateral distances [21]. Yet, other factors can induce the emergence of pinholes in the barriers, such as mechanical strain, defect propagation through the interface, and oxygen migration due to long time storage.

\section{Summary}

We have deposited, nanofabricated and measured allperovskite YBCO/LMO/YBCO S/FI/S tunnel junctions. The junction with a thicker FI barrier exhibited a large negative magnetoresistance below $T_{\text {Curie }}$, typical for colossal magnetoresistive manganites, but no supercurrent. Junctions with thin 5-nm LMO showed large supercurrent, but no signs of magnetism. Furthermore, the lack of a Fraunhofer modulation of the supercurrent indicated that it was caused by shorts in the barrier. Junctions with intermediate 10-nm LMO do show the desired behavior with significant (although much weaker than in 20-nm LMO) magnetism and signatures of Josephson current. The observed supercurrent in our S/FI/S junctions cannot be confidently ascribed to the spin-triplet component of the order parameter [1-3], because the latter requires a non-collinear magnetic state, which can be controllably generated only in spin-valve-type multilayer structures [4-6].

Acknowledgements We are thankful to I. Marozau for deposition of $\mathrm{YBCO} / \mathrm{LMO} / \mathrm{YBCO}$ trilayers. Technical support from the Core Facility in Nanotechnology at Stockholm University is gratefully acknowledged.

Funding Information This work was supported by the Swiss National Science Foundation Sinergia Grant No. CRSII2-154410/1 and No. 200020-172611, and the Swedish Foundation for International Cooperation in Research and Higher Education Grant No. IG20135453 .

Open Access This article is distributed under the terms of the Creative Commons Attribution 4.0 International License (http://creativecommons.org/licenses/by/4.0/), which permits unrestricted use, distribution, and reproduction in any medium, provided you give appropriate credit to the original author(s) and the source, provide a link to the Creative Commons license, and indicate if changes were made.

\section{References}

1. Buzdin, A.I.: Proximity effect in superconductor-ferromagnet heterostructures. Rev. Mod. Phys. 77, 935-976 (2005)

2. Bergeret, F.S., Volkov, A.F., Efetov, K.B.: Odd triplet superconductivity and related phenomena in superconductor-ferromagnet structures. Rev. Mod. Phys. 77, 1321-1373 (2005)

3. Asano, Y., Sawa, Y., Tanaka, Y., Golubov, A.A.: Odd-frequency pairs and Josephson current through a strong ferromagnet. Phys. Rev. B 76, 224525 (2007) 
4. Robinson, J.W., Witt, J.D.S., Blamire, M.G.: Controlled injection of Spin-Triplet supercurrents into a strong ferromagnet. Science 329, 59-61 (2010)

5. Khaire, T.S., Khasawneh, M.A., Pratt, W.P. Jr., Birge, N.O.: Observation of Spin-Triplet superconductivity in Co-Based Josephson junctions. Phys. Rev. Lett. 104, 137002 (2010)

6. Iovan, A., Golod, T., Krasnov, V.M.: Controllable generation of a spin-triplet supercurrent in a Josephson spin valve. Phys. Rev. B 90, 134514 (2014)

7. Keizer, R.S., Goennenwein, S.T.B., Klapwijk, T.M., Miao, G., Xiao, G., Gupta, A.: A spin-triplet supercurrent through the half-metallic ferromagnet $\mathrm{CrO}_{2}$. Nature 439, 825-827 (2006)

8. Singh, A., Jansen, C., Lahabi, K., Aarts, J.: High-quality $\mathrm{CrO}_{2}$ nanowires for dissipation-less spintronics. Phys. Rev. X 6, 041012 (2016)

9. Hu, T., Xiao, H., Visani, C., Sefrioui, Z., Santamaria, J., Almasan, C.C.: Evidence from MR for an induced triplet supercond. state in LMO/YBCO multilayers. Phys. Rev. B 80(R), 060506 (2009)

10. Dybko, K., Werner-Malento, K., Aleshkevych, P., Wojcik, M., Sawicki, M., Przyslupski, P.: Possible spin-triplet supercond. phase in the LMO/YBCO/LMO trilayer. Phys. Rev. B 80, 144504 (2009)

11. Kalcheim, Y., Kirzhner, T., Koren, G., Millo, O.: Longrange proximity effect in $\mathrm{La}_{2 / 3} \mathrm{Ca}_{1 / 3} \mathrm{MnO}_{3} /(100) \mathrm{YBa}_{2} \mathrm{Cu}_{3} \mathrm{O}_{7-\delta}$ ferromagnet/superconductor bilayer: Evidence for induced triplet superconductivity in the ferromagnet. Phys. Rev. B 83, 064510 (2011)

12. Visani, C., Sefrioui, Z., Tornos, J., Leon, C., Briatico, J., Bibes, M., Barthelemy, A., Santamaria, J., Villegas, J.E.: Equal-spin Andreev reflection and long-range coherent transport in high temperature superconductor/half-metallic ferromagnet junctions. Nature Phys. 8, 539 (2012)

13. Golod, T., et al.: High bias anomaly in $\mathrm{YBa}_{2} \mathrm{Cu}_{3} \mathrm{O}_{7-x} / \mathrm{LaMnO}_{3+\delta} /$ $\mathrm{YBa}_{2} \mathrm{Cu}_{3} \mathrm{O}_{7-x}$ superconductor/ferromagnetic insulator/superconductor junctions: Evidence for a long-range superconducting proximity effect through the conduction band of a ferromagnetic insulator. Phys. Rev. B 87, 134520 (2013)

14. Marozau, I., Das, P.T., Döbeli, M., Storey, J.G., Uribe-Laverde, M.A., Das, S., Wang, C., Rössle, M., Bernhard, C.: Influence of
$\mathrm{La}$ and $\mathrm{Mn}$ vacancies on the electronic and magnetic properties of $\mathrm{LaMnO}_{3}$ thin films grown by pulsed laser deposition. Phys. Rev. B 89, 174422 (2014)

15. Krasnov, V.M., Golod, T., Bauch, T., Delsing, P.: Anticorrelation between temperature and fluctuations of the switching current in moderately damped Josephson junctions. Phys. Rev. B 76, 224517 (2007)

16. Renshaw Wang, X., Li, C.J., Lü, W. M., Paudel, T.R., Leusink, D.P., Hoek, M., Poccia, N., Vailionis, A., Venkatesan, T., Coey, J.M.D., Ariando, Tsymbal, E.Y., Hilgenkamp, H.: Imaging and control of ferromagnetism in $\mathrm{LaMnO}_{3} / \mathrm{SrTiO}_{3}$ heterostructures. Science 349, 716-719 (2015)

17. Yamada, H., Xiang, P.-H., Akihito, S.: Phase evolution and critical behavior in strain-tuned $\mathrm{LaMnO}_{3}-\mathrm{SrMnO}_{3}$ superlattices. Phys. Rev. B 81, 014410 (2010)

18. Casanove, M.-J., Roucau, C., Baulès, P., Majimel, J., Ousset, J.C., Magnoux, D., Bobo, J.F.: Growth and relaxation mechanisms in $\mathrm{La}_{0.66} \mathrm{Sr}_{0.33} \mathrm{MnO}_{3}$ manganites deposited on $\mathrm{SrTiO}_{3}\left(\begin{array}{lll}0 & 0 & 1\end{array}\right)$ and $\mathrm{MgO}(000$ 1). Appl. Surf. Sci. 188, 19-23 (2002)

19. Wiedenhorst, B., Höfener, C., Lu, Y., Klein, J., Alff, L., Gross, R., Freitag, B.H., Mader, W.: Strain effects and microstructure of epitaxial manganite thin films and heterostructures. Appl. Phys. Lett. 74, 3636 (1999)

20. Siwach, P.K., Singh, H.K., Srivastava, O.N.: Influence of strain relaxation on magnetotransport properties of epitaxial $\mathrm{La}_{0.7} \mathrm{Ca}_{0.3} \mathrm{MnO}_{3}$ films. J. Phys.: Condens. Matter 18, 9783-9794 (2006)

21. Malik, V.K., Marozau, I., Das, S., Doggett, B., Satapathy, D.K., Uribe-Laverde, M.A., Biskup, N., Varela, M., Schneider, C.W., Marcelot, C., Stahn, J., Bernhard, C.: Pulsed laser deposition growth of heteroepitaxial $\mathrm{YBa}_{2} \mathrm{Cu}_{3} \mathrm{O}_{7} / \mathrm{La}_{0.67} \mathrm{Ca}_{0.33} \mathrm{MnO}_{3}$ superlattices on $\mathrm{NdGaO}_{3}$ and $\mathrm{Sr}_{0.7} \mathrm{La}_{0.3} \mathrm{Al}_{0.65} \mathrm{Ta}_{0.35} \mathrm{O}_{3}$ substrates. Phys. Rev. B 85, 054514 (2012)

Publisher's Note Springer Nature remains neutral with regard to jurisdictional claims in published maps and institutional affiliations. 